\title{
Research Progress of Computer in Medicine
}

\author{
Haidong Zhu' ${ }^{1, a}$, Jialin $\mathrm{Gao}^{1, \mathrm{~b},{ }^{*}}$ \\ ${ }^{1}$ Department of Urology, The First Hospital of Jilin University, Jilin, China

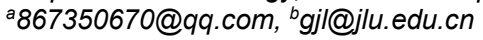

\begin{abstract}
With the rapid development of electronic computers, computer technology has been applied into various fields of medicine and its management. The application of computers in medical treatment has also greatly promoted the development of medical treatment. Research on the application condition of computers in medicine is of great importance to future medical care.
\end{abstract}

Keywords: Computer; Medicine.

The computer was firstly used in the field of basic medicine. Later, with the advancement of computer technology and medical research, computer and medicine become inseparable. In recent years, the application of computer technology in the field of medical information has become a hotspot of scientific research, having received widespread attention from the society.

\section{Application of computer in hospital information system}

The hospital information system is mainly for the prevention and treatment of various diseases and the provision of various medical treatment techniques for patients. In order to promote the development of hospital information systems, it is necessary to improve scientific research capabilities and technical level. To build a hospital's information system requires analyzing a large number of clinical data and pathology reports as well as integrated processing. At present, the hospitals, especially the well-known and influential large hospitals, not only have many internal institutions and personnel, but also need to face thousands of medical patients who come to the hospital every day, which require the hospital to pursue refined, error-free and standardized management. Computer information system has been widely used in hospitals in registration fees, diagnosis and hospitalization, and also in human resource management, medical care management and property management, thus improving the overall management and service level of the hospital. Besides, the computer information system also plays an indispensable role in the construction of hospital digital technology, the medical work and medical teaching of hospitals, the retrieval and sharing of medical resources information, and the implementation of centralized consultation, long-distance medical consultation and multimedia video guidance treatment.

\section{Application of computer in electronic medical record}

Electronic medical record is also called computerized medical record system or computer-based patient record. It is a digital medical record that is stored, managed, transmitted and reproduced by using electronic devices (computers, health cards, etc.), to replace handwritten paper medical record. Its content includes all the information of paper medical record. Medical record is to record the patient's disease and health status, which is a communication bridge between the hospital staff and the patient, and is the primary basis for doctors to develop medical plans. Electronic medical record has outstanding features: 1) convenient information browsing; 2) powerful storage capability; 3 ) realizing the sharing of medical record information. The use of electronic medical record system will greatly improve work efficiency, save a lot of valuable time for medical staff, and free medical staff from the heavy writing of various records, so that medical staff have more time to observe changes in patients' condition, better contact and communication with patients, and give more care and better treatment to patients, which is conducive to establishing a good doctor-patient relationship; meanwhile, they have more time for research activities, thus improving the level of medical technology. The use of the electronic medical record system also greatly improves the quality of the medical records of the hospital, thereby making the written medical records more standardized and more valuable for research and utilization. The management level of the hospital has reached a new level, for the 
management department can monitor and evaluate the work of each department based on this new management method of the management and assessment in the hospital, such as the amount of medical records, the ward rounds of the superior doctors and so on. The use of electronic medical records can speed up the flow of patient information, so that patient information can be obtained anywhere at any time, which is the service that paper medical records cannot provide. The use of the electronic medical record system makes the medical records paperless, saves hospital expenses, reduces operating costs, and improves economic efficiency [1].

\section{Application of computer complementary medicine}

in

Medical images with different functions are divided into two types, structural image technology and functional image technology. The former is mainly used to obtain images of anatomical structures of various organs in the human body. With such structure perspective images, medical personnel can diagnose human organic diseases without anatomical examination. CT and MRI are the representatives of such structural images. However, when the human organs have early lesions, although the organ structure is still normal, some physiological functions of the organs, such as metabolism, begin to change abnormally. At this time, using structural image to conduct structural anatomical examination is impossible to diagnose the diseased organs in time, and it is necessary to use functional image technology based on SPECT and PET. Functional image can detect changes in biochemical activity of human organs and present them as functional images [2]. The computer has powerful programmatic analysis function, which can quickly analyze more complex medical images, such as B-ultrasound, X-ray, and MRI. The main applications are listed as follows. 1) Compress, convert and deform medical images; 2) Analyze the calculation of mathematical formulas in the image; 3) Realize the mutual conversion of images and numbers; 4) Simulate and restore the blurred or defective images through a computer; 5) Decompose complex medical images to facilitate doctors to conduct more detailed analysis and research and to detect blurred corners; 6) The computer compresses large medical images through different image processing software for convenient storage and transmission. Computer is an important tool and means to improve the quality of education and cultivate more high-level talents [3]. At the same time, computer also plays a major role in medical laboratory science. 1) Electromechanical control and maintenance and inspection management of medical inspection instruments. Almost all automatic inspection instruments are inseparable from the microcomputer. The program in the industrial single-board machine memory can accurately control the various instrument movements of the equipment. This curing program is generally unchangeable and can be used alone or communicate with the PC connected to the instrument. 2) Measure and calculate at the right time. Due to the huge computing power of computers, the era of manual drawing and manual calculations has gone forever. With the excellent computing power and improved algorithm of the computer, the improved enzyme rate method and search enzyme rate method are realized. These methods can conduct optimal calculation according to the measurement environment, conditions, reagent characteristics and sample content, and also can draw various spline curves, greatly improving measurement accuracy and sensitivity. Biochemical indicators, automatic analysis of physiological information and intelligentization of medical equipment. Intelligentization of medical equipment refers to the combination of modern medical instruments and computer technology and various software, which enables these devices to have the functions of automatic sampling, automatic analysis and automatic data processing, and real-time control can be achieved. It is one direction of the development for medical instruments. 3) Data processing, integration and management. The importance of post-processing of data has gained increasingly more attention with the development and application of statistics in the field of medical research. The seemingly chaotic data contains infinite scientific laws and mysteries. Through the collation and refinement of clinical test data, and with relevant statistical software, we can find out the rules [4].

\section{Application of computer in nursing work}

Nursing data are the true record of nursing work, with the value of research, inspection and promoting nursing quality. With the deepening implementation of hierarchical management in hospitals, the requirements for hierarchical management in hospitals are getting higher and higher. How to do a good job in nursing data is a problem faced by all nursing management workers. Computers are widely used in the field of nursing. Many hospitals have formed a complete microcomputer network information management system, and gradually realize the standardization, integration, networking and informationization of nursing work, promoting the nursing work to a new development stage. The applications of computer in nursing work are mainly divided into the following three aspects, (1) nursing, including nursing records, nursing examination, patient monitoring, drug management, etc.; (2) nurse education, including nursing CAI education, nurse teaching plan and academic record management; (3) nurse management, including nurse service plan scheduling, human resource management, inspection or evaluation of nurses' work quality. The benefit brought by the application of computers to nursing work is that using Skynet system to process doctor's advice can make the record and management of nursing documents gradually scientific, systematic and standardized, which is conducive to the permanent preservation of nursing materials and the nursing office automation, thus reducing errors. Apply computerized multi-care to conduct lectures, examinations and evaluations: the nursing department can design a system with lectures, examinations and 
evaluations according to the actual situation. The system includes flip-through function, lecture function and review and assessment function [5].

Conclusion

At present, the application of computer technology in the medical field in China is increasingly more perfect. As an important part of the development of medicine, computer technology will definitely continue to promote the rapid development of medical technology in the future. In short, computer technology has achieved certain results in medical applications currently. With the advancement and development of science and technology, computer technology and medical technology will surely usher in a new situation, and they will be further combined and continuously promoted.

\section{References}

1. Li Lei. Application of Computer Technology in Medicine [J]. Nanfeng, 2016, (5): 148

2. Bu Jinmei, Xiao Yi, Long Mei, et al. Application of Computer in Medical Image Processing [J]. China Medical Education Technology, 2001, (4): 203-204.

3. Liu Xiling. Computer Aided System. Beijing: Tsinghua University Press, 1993, 105.

4. Liu Gang. Application Evaluation of Computer and Network Technology in Medical Examination [J]. Journal of Huaihai Medicine, 2003, 21 (4): 342-343.

5. Yang Rong, Huang Yan. Application of Computer in Nursing Work [J]. Chinese Community Doctors (Medical Speciality), 2011, (31): 359. 